\title{
Reduction of the phase jitter in differential phase-shift-keying soliton transmission systems by in-line Butterworth filters
}

\author{
S. A. Derevyanko* and S. K. Turitsyn \\ Photonics Research Group, Aston University, Birmingham B4 7ET, UK
}

Received August 25, 2003

\begin{abstract}
We examine reduction of phase jitter by use of in-line Butterworth filters in soliton systems in the context of differential phase-shift-keying coding. We also demonstrate numerically that the use of a Butterworth filter in a return-to-zero differential phase-shift-keying system can reduce continuum background radiation. (C) 2004 Optical Society of America

OCIS codes: $060.2330,190.5530$
\end{abstract}

Recent progress in the practical implementation of the return-to-zero (RZ) differential phase-shift-keying (DPSK) modulation format in fiber communication ${ }^{1}$ has rekindled interest in the statistics of the phase of an optical pulse propagating along the transmission line. The effect of filter control on the phase jitter in phase-shift keying systems has been analyzed in Refs. 2 and 3. In systems without filtering, self-phase modulation causes strong dynamic coupling between the amplitude and phase fluctuations, which was first pointed out in Ref. 4. Such a coupling causes the variance of the phase jitter to grow proportionally to the cube of the propagation distance, which is similar to the Gordon-Haus effect for the soliton timing position. The use of filters reduces the random walk of the soliton frequency and amplitude, and, as a result, in a system with in-line filtering, phase fluctuations grow only linearly with distance., ${ }^{2,3}$ This provides a completely different scale for error-free propagation distances and sets the scene for the RZ DPSK transmission, in which the logical bits are coded by the carrier pulse relative phases. The study of the statistics of DPSK systems has recently attracted a good deal of interest. ${ }^{3,5-7}$

In Ref. 8 the use of in-line Butterworth filters (BFs) that can combat frequency and amplitude jitters more efficiently than the usual shallow filters was proposed. More important, BFs reduce the growth rate for the radiative background generated because of the applied extra gain. The reduction of the amplitude jitter by use of BFs must also have a significant effect on the phase jitter. Recent advances in fiber-optic technologies offer new possibilities for the fabrication of sophisticated system components. In particular, fiber grating technology is well adapted to making in-line flat-top filters such as $\mathrm{BF}$ with controlled wavelength-dependent finesse. In this Letter we examine the effect of BF-based soliton control on pulse statistics and demonstrate how the phase jitter is suppressed by use of in-line flat-top BFs.

The path-averaged optical signal propagation under the combined action of the in-line BFs and the additive white Gaussian noise is described by the following equation (presented in soliton units) ${ }^{8}$ :

$$
\frac{\partial u}{\partial z}=\frac{i}{2} \frac{\partial^{2} u}{\partial t^{2}}+i|u|^{2} u+\frac{1}{2}\left[\alpha-\eta_{n}\left(i \frac{\partial}{\partial t}\right)^{2 n}\right] u+n,
$$

where $2 n$ is the order of the filter, $\eta_{n}$ is the filter strength, and $\alpha$ is an excess gain to compensate for the filter loss. Additive white Gaussian noise is accounted for by the term $n$, which has the following statistics:

$$
\begin{aligned}
\langle n(t, z)\rangle & =\left\langle n(t, z) n\left(t^{\prime}, z^{\prime}\right)\right\rangle=0, \\
\left\langle n(t, z) n^{*}\left(t^{\prime}, z^{\prime}\right)\right\rangle & =D \delta\left(z-z^{\prime}\right) \delta\left(t-t^{\prime}\right) .
\end{aligned}
$$

Here $D$ is the path-averaged amplified spontaneous emission noise power in soliton units (see Ref. 2).

Both noise and filter action can be treated as perturbations. Applying standard perturbation theory (see, for instance, Refs. 2 and 8-10) to a single soliton ansatz,

$$
\begin{aligned}
u_{0}(t, z)= & A(z) \operatorname{sech}\{A(z)[t-T(z)]\} \\
& \times \exp [-i \Omega(z) t+i \phi(z)],
\end{aligned}
$$

one can derive the system of Langevin equations with multiplicative noise for the soliton parameters. ${ }^{2}$ For such a system it is possible to write (details will be published elsewhere) a Fokker-Planck equation (FPE) for the joint probability density function (PDF) $P(A, \phi, \Omega, T \mid z)$ (see, for instance, Ref. 11):

$$
\begin{aligned}
& \frac{\partial P}{\partial z}=\left[-\frac{1}{2}\left(A^{2}-\Omega^{2}\right)+\eta_{n} T \sum_{j=0}^{n-1}\left(\begin{array}{c}
2 n \\
2 j+1
\end{array}\right) M_{n-j} \Omega^{2 j+1}\right. \\
& \left.\times A^{2(n-j}\right] \frac{\partial P}{\partial \phi}+\Omega \frac{\partial P}{\partial T}-\frac{\partial}{\partial A}\left[D P+\alpha A P-\eta_{n} \sum_{j=0}^{n}\left(\begin{array}{c}
2 n \\
2 j
\end{array}\right)\right. \\
& \left.\times M_{n-j} \Omega^{2 j} A^{2(n-j)+1} P\right]+\frac{\partial}{\partial \Omega}\left[\eta_{n} \sum_{j=0}^{n-1}\left(\begin{array}{c}
2 n \\
2 j+1
\end{array}\right) M_{n-j} \Omega^{2 j+1}\right. \\
& \left.\times A^{2(n-j)} P\right]+D\left[\frac{1}{6} A T^{2}+\frac{1}{12 A}\left(2+\frac{\pi^{2}}{6}\right)\right] \frac{\partial^{2} P}{\partial \phi^{2}}+\frac{D}{3} A T \\
& \times \frac{\partial^{2} P}{\partial \phi \partial \Omega}+\frac{D}{2} \frac{\partial^{2}}{\partial A^{2}}(A P)+\frac{D}{6} A \frac{\partial^{2} P}{\partial \Omega^{2}}+\frac{D \pi^{2}}{24 A^{3}} \frac{\partial^{2} P}{\partial T^{2}} .
\end{aligned}
$$


Here $M_{n}$ are the $2 n$th derivatives of the generating function $s / \sin (s)$ calculated at $s=0$ (see Ref. 8). At the origin $z=0$ soliton parameters have deterministic values so that $P(A, \phi, \Omega, \phi \mid 0)=$ $\delta\left(A-A_{0}\right) \delta\left(\phi-\phi_{0}\right) \delta\left(T-T_{0}\right) \delta\left(\Omega-\Omega_{0}\right)$, where index 0 stands for the initial value of the corresponding soliton parameter. Equation (5) describes the statistics (not necessarily Gaussian) of a single soliton transmission in the presence of filters. When the deviations of parameters from the initial values are small, one can substitute the diffusion coefficients in the terms with second derivatives in Eq. (5) for their initial values, since those coefficients are fairly smooth functions of soliton parameters, whereas the PDF can remain a sharp function. The same procedure applies to the drift coefficients in the terms with first derivatives, but while approximating those coefficients we must keep linear terms such as $\Omega-\Omega_{0}$ as well. In this approximation the statistics of soliton parameters appear to be Gaussian, which justifies the results obtained in Refs. 3 and 5-7. In the remaining part of this Letter we focus on the analysis of the phase jitter in the presence of flat-top filters.

The initial soliton parameters in the FPE correspond to stable stationary propagation in a system without noise. Before we proceed, it is convenient to redefine phase $\phi$ to include the deterministic part of the nonlinear phase modulation: $\quad \phi \rightarrow\left[\phi-(1 / 2) A_{0}^{2} z\right] \bmod 2 \pi$. To ensure the stability of the pulse propagation we must choose the initial values of the soliton parameters to equal their stationary values. The stationary values of soliton parameters can be found as stationary characteristics of Eq. (5) under the condition $D=0$.

A simple analysis shows that the stationary solution for frequency $\Omega_{0}=\Omega_{s}$ is zero, the stationary phase $\phi_{0}=\phi_{s}$ and the timing position $T_{0}=T_{s}$ are arbitrary constants (of course, $0 \leq \phi_{0}<2 \pi$ ), and the stationary amplitude is defined by the following relation between the $\mathrm{BF}$ characteristics and the excess gain (see Ref. 8):

$$
\alpha=\eta_{n} A_{s}^{2 n} M_{n}=\eta_{n} A_{0}^{2 n} M_{n} .
$$

Therefore, without loss of generality in what follows we assume that the initial values of frequency $\Omega_{0}$ and soliton position $T_{0}$ are equal to zero. After we apply the linearization procedure described above to Eq. (5), we arrive at the FPE for the Ornstein-Ulenbeck process in which the diffusion coefficients are constants while the drift coefficients are linear functions of variables $A-A_{0}, \phi-\phi_{0}, \Omega$, and $T$ (see, for instance, Ref. 11). In this Letter we are primarily interested in phase jitter, since it is the most important for the DPSK system. Therefore we can use the well-known Gaussian solution for the Ornstein-Ulenbeck process and establish the variance of the phase jitter as

$$
\begin{aligned}
\left\langle\delta \phi^{2}(z)\right\rangle= & \frac{D A_{0}^{3}}{16 n^{3} \alpha^{3}}[-3+4 n \alpha z \\
& +4 \exp (-2 n \alpha z)-\exp (-4 n \alpha z)] \\
& +\frac{D}{3 A_{0}}\left(1+\frac{\pi^{2}}{12}\right) z
\end{aligned}
$$

First, note that using $A_{0}=1$ and $n=1$ (Gaussian filter) in Eq. (7) yields the result derived in Ref. 3. In the presence of filters the phase jitter grows only linearly with distance, whereas without filtering the phase jitter increases as $z^{3}$. In what follows we compare our results with the phase jitter calculated in Ref. 3. For the system described in Ref. $3, A_{0}=1, n=1$, and $\eta=0.249$, which yields $\alpha=0.083$. The effective dimensionless noise strength used in Ref. 3 is $D=2.9 \times 10^{-5}$. For $z=8,000 \mathrm{~km}$ this corresponds to the standard deviation of $\Delta=\left(\left\langle\delta \phi^{2}\right\rangle\right)^{1 / 2} / \pi=\Delta_{\text {et }}=0.099$. With BFs we have an extra parameter $n$ that actually allows us to control the suppression of the jitter. From Eq. (7) one can see that for large propagational distances $z$ the variance $\left\langle\delta \phi^{2}\right\rangle$ grows linearly in $z$, and the rate of growth is smaller for higher orders of the $\mathrm{BF}$. Therefore the use of BFs significantly reduces phase jitter compared with the case of the simple Gaussian filter considered in Ref. 3. The higher the filter order, the better the improvement.

Next we examine the possibility of minimizing phase jitter by varying the filtering characteristics. Let us fix the propagation distance $z$. Then the variance $\left\langle\delta \phi^{2}\right\rangle$ is a function of four parameters: excess gain $\alpha$, filter strength $\eta$, filter order $2 n$, and soliton amplitude $A_{0}$, which is related to the soliton power and pulse width. However, these parameters are not independent-Eq. (6) imposes an obvious constraint. Also, because the filters are accounted for in a perturbative manner, the values $\alpha$ and $\eta$ must not be large. The initial amplitude of the soliton, $A_{0}$, is also restricted in some range by the power constraints and the bit rate. Taking this into account, for each value of $n$ we express $A_{0}$ as a function of $\eta$ for fixed $\alpha$. Inserting $A_{0}(\eta)$ into Eq. (7), we note that $\left\langle\delta \phi^{2}\right\rangle$ is a nonmonotonic function of $\eta$-there exists an optimal value of filter strength $\eta_{\text {opt }}$ that provides a minimum for the phase jitter $\left\langle\delta \phi^{2}\right\rangle$ for the given $n$ and $\alpha$. One can easily find this extremum and calculate the optimal values of the parameters. For long-haul transmission systems we are interested in distances for which the inequality $\alpha n z \gg 1$ holds so that we can neglect the exponents in the first term on the right-hand side of Eq. (7). Then the optimal value of $\eta$ and the corresponding jitter are given by

$$
\begin{aligned}
\eta_{\mathrm{opt}}(n, \alpha) & =\left[\frac{9}{n^{2}\left(4+\pi^{2} / 3\right)}\right]^{n / 2} \frac{\alpha^{1-n}}{M_{n}}, \\
\left\langle\delta \phi_{\mathrm{opt}}^{2}\right\rangle & =\gamma \frac{D z}{n^{1 / 2} \alpha^{1 / 2}},
\end{aligned}
$$

where $\gamma=2^{-1 / 2}\left(3^{-1 / 2}+3^{-3 / 2}\right)\left(1+\pi^{2} / 12\right)^{3 / 4} \approx 0.854$ is a numerical factor. In Fig. 1 we plot the relative optimum standard deviation $\Delta / \Delta_{\text {et }}$ versus the excess gain $\alpha$. Again we see that the use of BFs may reduce phase jitter by at least five times, provided that the system parameters have been optimized properly. The physical reason for this is that for the fixed value of the excess gain, $\alpha$, the effective restoring force that shifts the soliton back to the unperturbed position [the components of this force are given by the drift coefficients 


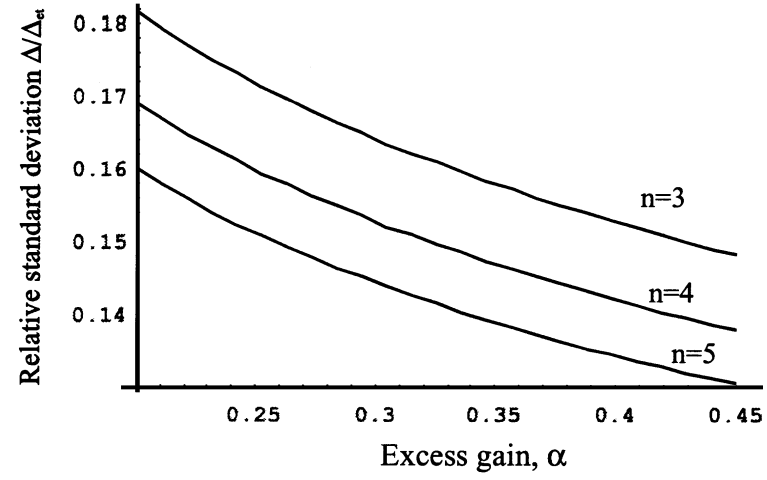

Fig. 1. Optimal relative standard deviation of the soliton phase, $\Delta / \Delta_{\text {et }}$, plotted versus the excess gain $\alpha$ for different values of filter order $2 n$.

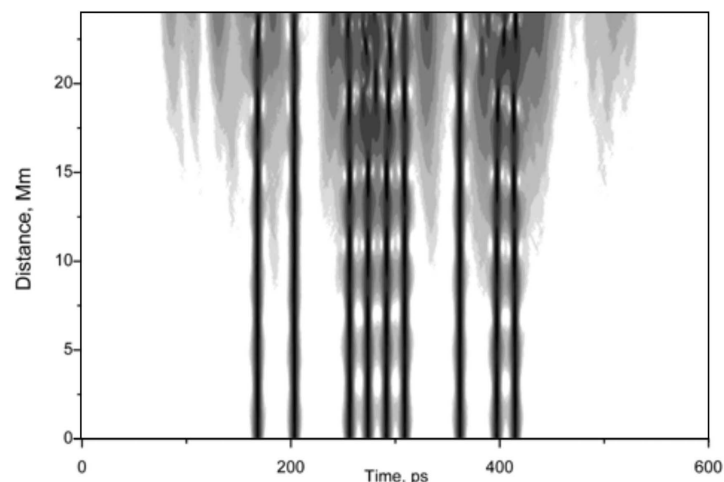

(a)

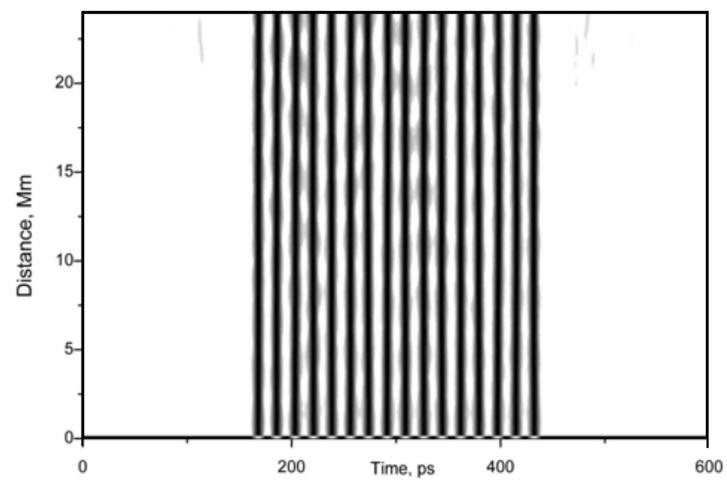

(b)

Fig. 2. Logarithm of the field intensity plotted as a density plot. The result is an intensity profile for 16 pseudorandom bits, averaged over 100 samples of the amplified spontaneous emission noise. (a) RZ intensity modulation format with conventional filters $(n=1)$. (b) Same bit pattern as in (a) but for the RZ DPSK format with high-order Butterworth filters $(n=3)$.

in Eq. (5)], is $n$ times bigger than that for the etalon system with $n=1$. A simple analysis of Eqs. (8) and (9) shows that for high-order filters $(n \geq 2)$ it is possible to reduce phase jitter by at least five times compared with the system using conventional filters, with the filter strength and excess gain still remaining relatively small.
Equation (9) shows that the minimal value of the phase jitter decreases with the increase in the filter order $n$. The increase in the excess gain $\alpha$ that formally also leads to jitter reduction is limited by the constraint of the smallness of $\alpha$. Additionally, the increase in the excess gain is a trade-off between the jitter reduction and the growth rate of the radiative background between the solitons. Note, however, that in the RZ DPSK systems there are no empty slots between the pulses, and therefore the background generation should be considerably suppressed. To illustrate this, in Fig. 2 we provide the results of numerical simulations of the propagation of a pseudorandom pattern of 16 bits. One can see that the use of RZ DPSK [Fig. 2(b)] is much more beneficial in terms of suppression of the continuum than a conventional filter of the same strength [Fig. 2(a)].

In conclusion, we have examined the reduction of the phase jitter by use of in-line Butterworth filters. Flat-top in-line filters can be especially beneficial for the RZ DPSK systems for which the radiative background generation is weaker than that for the traditional RZ formats. This provides for the important possibility of a trade-off between phase jitter reduction and generation of a radiative background caused by filtering.

S. Derevyanko acknowledges an Intas Young Scientist Fellowship YS 2002-165. This work was also supported by the Leverhulme Trust Project F/00250/ B. S. Derevyanko's e-mail address is s.derevyanko@ aston.ac.uk.

*Also with the Institute for Radiophysics and Electronics of the National Academy of Sciences of Ukraine, Kharkov, 61085 Ukraine.

\section{References}

1. A. H. Gnauck, G. Raybon, S. Chandrasekhar, J. Leuthold, C. Doerr, L. Stulz, A. Agarwal, S. Banerjee, D. Grosz, S. Hunsche, A. Kung, A. Marhelyuk, D. Maywar, M. Movassaghi, X. Liu, C. Xu, X. Wei, and D. M. Gill, in Optical Fiber Communication Conference (OFC), Vol. 70 of OSA Trends in Optics and Photonics Series (Optical Society of America, Washington, D.C., 2002), postdeadline paper FC2.

2. E. Iannoe, F. Matera, A. Mecozzi, and M. Settembre, Nonlinear Optical Communication Networks (Wiley, New York, 1998).

3. M. Hanna, H. Porte, J. P. Goedgebuer, and W. T. Rhodes, Opt. Lett. 24, 732 (1999).

4. J. P. Gordon and L. F. Mollenauer, Opt. Lett. 15, 1351 (1990).

5. M. Hanna, H. Porte, J. P. Goedgebuer, and W. T. Rhodes, IEEE J. Quantum Electron. 36, 1333 (2000).

6. M. Hanna, H. Porte, J. P. Goedgebuer, and W. T. Rhodes, Electron. Lett. 37, 644 (2001).

7. C. J. McKinstrie and C. Xie, IEEE J. Sel. Top. Quantum Electron. 8, 616 (2002).

8. A. Mecozzi, Opt. Lett. 20, 1859 (1995).

9. D. J. Kaup, Phys. Rev. A 42, 5689 (1990).

10. H. A. Haus and Y. Lai, J. Opt. Soc. Am. B 7, 386 (1990).

11. H. Risken, The Fokker-Planck Equation (Springer, New York, 1996). 